MATHEMATICS OF COMPUTATION

Volume 68, Number 226, April 1999, Pages 547-557

S 0025-5718(99)01022-4

\title{
ON THE CONVERGENCE OF BOUNDARY ELEMENT METHODS FOR INITIAL -NEUMANN PROBLEMS FOR THE HEAT EQUATION
}

\author{
YANG HONGTAO
}

\begin{abstract}
In this paper we study boundary element methods for initialNeumann problems for the heat equation. Error estimates for some fully discrete methods are established. Numerical examples are presented.
\end{abstract}

\section{INTRODUCTION}

Boundary element methods have been applied to initial boundary value problems for the heat equation (IBVPHE) (see [4], [5], [8], [15], [20]). The boundary integral equations (BIE) derived by using the potential method or the Green's formula method are Volterra-Fredholm BIEs of the first kind (V-FBIE1) with weakly or strongly singular kernels, or of the second kind (V-FBIE2) with weakly singular kernels. Following the usual numerical treatment of parabolic problems (see [11]), we can discretize the space variables and the time variable together for solving these V-FBIEs numerically. This approach may be the Galerkin method, the collocation method, etc. We can also discretize the space variables and the time variable separately, which is more flexible in the design of algorithms. For example, one may discretize the space variables by using the Galerkin method, the collocation method, or the Petrov-Galerkin method, and then solve the Volterra system of the time variable by using the Galerkin method, the collocation method, or the product integral method. This approach is employed in this paper.

The mathematical theory of boundary element methods for the IBVPHE has been developed only in recent years. Error analysis of the Galerkin method for V-FBIEs has been carried out (see [1], [6], [17]). Convergence and stability of the collocation method for V-FBIE2 have been established in [18] for the Neumann problem of the heat equation on convex domains. As the further work of [18], the projection method for V-FBIE2 in the space of continuous functions are studied in [7], [19] for non-smooth domains.

In this paper, we consider the initial-Neumann problem for the heat equation (INPHE). Here the solution of INPHE is represented by a single heat potential, and thus the unknown density function is the solution of a V-FBIE2 with a weakly singular kernel. The right side of the V-FBIE2 is a suitably smooth function or the singularity $O\left(t^{-1 / 2}\right)$ as $t \rightarrow 0^{+}$. In [21] we have established the well-posedness

Received by the editor January 4, 1994 and, in revised form, January 26, 1996 and February $18,1997$.

1991 Mathematics Subject Classification. Primary 65M30; Secondary 65R20.

Key words and phrases. Heat equation, boundary element method, error estimate.

This work was supported by the National Natural Science Foundation of China. 
theories for the V-FBIE2. Since the kernel has a singularity, the solution of VFBIE2 may not be smooth even if its right hand side is smooth. Therefore, we shall make proper variable transformations for the V-FBIE2 according to the properties of its right hand side, and we solve the new V-FBIE2s instead of the original density function.

The paper is organized as follows. In Section 2 we recall some of our previous results found in [21]. In Section 3 we construct some fully discrete methods for VFBIE2s. In Section 4 we derive the error estimates. Finally, in Section 5, some numerical results are presented.

For the sake of convenience, we introduce the following notations from [21]. Let $\Omega$ be a bounded domain in $R^{n}(n \geq 2)$ with the smooth boundary $\Gamma$ and $0<T<\infty$. For $m, k \in Z^{+}$(the non-negative integer set), denote

$$
C_{E}^{k}\left([0, T] ; C^{m}(\Gamma)\right)=\left\{\varphi \in C^{k}\left([0, T] ; C^{m}(\Gamma)\right): \varphi^{(j)}(0)=0, j=0,1, \ldots, k-1\right\},
$$

where $C^{k}\left([0, T] ; C^{m}(\Gamma)\right)$ is the space consisting of functions from $[0, T]$ to $C^{m}(\Gamma)$ which are $k$-times continuously differentiable (see [14]). Here $C^{m}(\Gamma)$ is the space of $m$-times continuously differentiable functions on $\Gamma$ with norm $\|\cdot\|_{m, \infty}$. For $\varphi \in C^{k}\left([0, T] ; C^{m}(\Gamma)\right)$, we define the norm as follows:

$$
\|\varphi\|_{m, k}=\max _{0 \leq j \leq k} \sup _{0 \leq t \leq T}\left\|\varphi^{(j)}(t)\right\|_{m, \infty}
$$

Equipped with this norm, $C^{k}\left([0, T] ; C^{m}(\Gamma)\right)$ is a Banach space with a closed subspace $C_{E}^{k}\left([0, T] ; C^{m}(\Gamma)\right)$. Similarly, we define the Banach space $C^{k}\left([0, T] ; H^{s}(\Gamma)\right)$ $\left(k \in Z^{+}, s \in R\right)$ and its closed subspace $C_{E}^{k}\left([0, T] ; H^{s}(\Gamma)\right)$. The norm for these two spaces is

$$
\|\varphi\|_{s, k, \infty}=\max _{0 \leq j \leq k} \sup _{0 \leq t \leq T}\left\|\varphi^{(j)}(t)\right\|_{s},
$$

where $\|\cdot\|_{s}$ stands for the norm of Sobolev space $H^{s}(\Gamma)$.

\section{Some RESUlts of $[21]$}

Consider the following initial-Neumann problem for the heat equation:

$$
\begin{aligned}
\frac{\partial u(t, x)}{\partial t} & =\Delta u(t, x)+f(t, x), \quad(t, x) \in(0, T] \times \Omega, \\
u(0, x) & =u_{0}(x), \quad x \in \Omega, \\
\frac{\partial u(t, x)}{\partial n(x)} & =b(t, x), \quad(t, x) \in(0, T] \times \Gamma,
\end{aligned}
$$

where $n(x)$ is the unit outward normal vector to $\Gamma$ at $x, f(t, x) \in C^{0}([0, T] \times \Omega)$ is locally Hölder continuous in $x$ uniformly with respect to $t, u_{0} \in C^{0}(\Omega), b \in$ $C^{0}([0, T] \times \Gamma)$. It is well known that the solution of this problem can be represented in the single heat potential form (see [10]):

$$
u(t, x)=\int_{0}^{t} \int_{\Gamma} G(t-\tau, x-y) \varphi(\tau, y) d s(y) d \tau+w(t, x), \quad(t, x) \in(0, T] \times \Omega,
$$

where $\varphi(t, x)$ is the unknown density function, $G(t, x)=(4 \pi t)^{-n / 2} \exp \left(-|x|^{2} / 4 t\right) H(t)$ $(H(t)$ is the Heaviside function) is the fundamental solution of the heat operator 
$\frac{\partial}{\partial t}-\Delta$, and

$$
\begin{aligned}
w(t, x)= & \int_{\Omega} G(t, x-y) u_{0}(y) d y \\
& +\int_{0}^{t} \int_{\Omega} G(t-\tau, x-y) f(\tau, y) d y d \tau,(t, x) \in(0, T] \times \Omega .
\end{aligned}
$$

And $\varphi$ satisfies the following Volterra-Fredholm boundary integral equation of the second kind:

$$
\begin{array}{r}
\frac{1}{2} \varphi(t, x)+\int_{0}^{t} \int_{\Gamma} \frac{\partial G(t-\tau, x-y)}{\partial n(x)} \varphi(\tau, y) d s(y) d \tau=g(t, x) \\
(t, x) \in(0, T] \times \Gamma
\end{array}
$$

where

$$
g(t, x)=b(t, x)-\int_{\Omega} \frac{\partial G(t, x-y)}{\partial n(x)} u_{0}(y) d y-\int_{0}^{t} \int_{\Omega} \frac{\partial G(t-\tau, x-y)}{\partial n(x)} f(\tau, y) d y d \tau .
$$

As shown in [21], $g(t, x)$ is suitably smooth at $t=0$ or has a singularity $O\left(t^{-1 / 2}\right)$ as $t \rightarrow 0^{+}$. In the latter case, we may assume that $t g\left(t^{2}, x\right)$ is sufficiently smooth at $t=0$. We further define $\psi(t, x)=t \varphi\left(t^{2}, x\right)$ and rewrite (2.4) as

$$
\begin{array}{r}
\frac{1}{2} \psi(t, x)+\int_{0}^{t} \int_{\Gamma} 2 t \frac{\partial G\left(t^{2}-\tau^{2}, x-y\right)}{\partial n(x)} \psi(\tau, y) d s(y) d \tau=\operatorname{tg}\left(t^{2}, x\right), \\
(t, x) \in\left[0, T^{1 / 2}\right] \times \Gamma .
\end{array}
$$

For $t>0$, define the following integral operator:

$$
K(t) q(x)=2 t^{1 / 2} \int_{\Gamma} \frac{\partial G(t, x-y)}{\partial n(x)} q(y) d s(y) .
$$

Then (2.4) and (2.5) can be rewritten respectively as

$$
\begin{gathered}
\varphi(t)+\int_{0}^{t}(t-\tau)^{-1 / 2} K(t-\tau) \varphi(\tau) d \tau=2 g(t), \quad t \in[0, T], \\
\psi(t)+\int_{0}^{t} 2 t\left(t^{2}-\tau^{2}\right)^{-1 / 2} K\left(t^{2}-\tau^{2}\right) \psi(\tau) d \tau=2 t g\left(t^{2}\right), \quad t \in\left[0, T^{1 / 2}\right],
\end{gathered}
$$

where $\varphi(t), \psi(t)$ and $h(t)$ denote the abstract functions of $t$ whose values are $\varphi(t, x), \psi(t, x)$ and $h(t, x)$, respectively. We have the following results for the properties of $K(t)$ (see Lemma 2.1-2.2 of [21]).

Theorem 2.1. Let $m, k \in Z^{+}, s \in R, q \in H^{2 k+s}(\Gamma)$ or $C^{2 k+m}(\Gamma)$. Then $\lim _{t \rightarrow 0^{+}} K^{(k)}(t) q$ exists in $H^{s}(\Gamma)$ or $C^{m}(\Gamma)$ and

or

$$
\left\|K^{(k)}(t) q\right\|_{s} \leq C\|q\|_{2 k+s}, \quad \forall t>0
$$

$$
\left\|K^{(k)}(t) q\right\|_{m, \infty} \leq C\|q\|_{2 k+m, \infty}, \quad \forall t>0
$$

where

$$
K^{(k)}(t) q(x)=\frac{d^{k} K(t) q}{d t^{k}}=\int_{\Gamma} \frac{\partial^{k}}{\partial t^{k}}\left(\frac{2 t^{1 / 2} \partial G(t, x-y)}{\partial n(x)}\right) q(y) d s(y),
$$


$C$ is a positive constant independent of $t, q$ (throughout this paper we shall denote a generic positive constant by $C$ ).

Remark 2.1. We can also show that $K(t)$ is a linear bounded operator from $L^{\infty}(\Gamma)$ to $C^{0}(\Gamma)$, and

$$
\|K(t) q\|_{0, \infty} \leq C\|q\|_{0, \infty}, \quad \forall t>0, q \in L^{\infty}(\Gamma),
$$

where $\|\cdot\|_{0, \infty}$ also denotes the norm of $L^{\infty}(\Gamma)$.

With the help of this theorem, we can prove that $\varphi \in C_{E}^{k}\left([0, T] ; H^{s}(\Gamma)\right)$ or $C_{E}^{k}\left([0, T] ; C^{m}(\Gamma)\right)$ and $\psi \in C^{k}\left([0, T] ; H^{s}(\Gamma)\right)$ or $C^{k}\left([0, T] ; C^{m}(\Gamma)\right)$ under some conditions (see Theorem 4.1 and 4.3 of $[21]$ ). When $g \in C^{k}\left([0, T] ; H^{s}(\Gamma)\right.$ ) (or $\left.C^{k}\left([0, T] ; C^{m}(\Gamma)\right)\right)$ but $g(0) \neq 0$, we only have that $\varphi\left(t^{2}\right) \in C^{k}\left([0, T] ; H^{s}(\Gamma)\right.$ ) (or $C^{k}\left([0, T] ; C^{m}(\Gamma)\right)$ ), while $\varphi(t)$ is only continuous at $t=0$ (see Theorem 4.2 of [21]). In this case, we let $\sigma(t)=\varphi\left(t^{2}\right)$ and rewrite (2.6) as

$$
\sigma(t)+\int_{0}^{t} 2 \tau\left(t^{2}-\tau^{2}\right)^{-1 / 2} K\left(t^{2}-\tau^{2}\right) \sigma(\tau) d \tau=2 g\left(t^{2}\right), \quad t \in\left[0, T^{1 / 2}\right] .
$$

\section{Product integration methods}

In order to find the solution $u(t, x)$ of $(2.1)-(2.3)$ numerically, we shall solve (2.6), (2.7) or (2.8) according to the property of $g(t)$ or the smoothness of $\varphi(t)$. Now we state these equations in the following general form:

$$
\phi(t)+\int_{0}^{t} p(t, \tau) B(t, \tau) \phi(\tau) d \tau=f(t), \quad t \in[0, \bar{T}],
$$

where $p(t, \tau)=(t-\tau)^{-1 / 2}, 2 t\left(t^{2}-\tau^{2}\right)^{-1 / 2}$, or $2 \tau\left(t^{2}-\tau^{2}\right)^{-1 / 2}, B(t, \tau)=K(t-\tau)$ or $K\left(t^{2}-\tau^{2}\right), f(t)=2 g(t), 2 t g\left(t^{2}\right)$, or $2 g\left(t^{2}\right), \bar{T}=T$ or $T^{-1 / 2}$.

Let $V$ be $H^{s}(\Gamma)$ or $C^{0}(\Gamma), V_{h}(0<h<1)$ be a family of boundary element spaces and $P_{h}(0<h<1)$ be linear operators mapping $V$ onto $V_{h}$. Then the semi-discrete method for equation (3.1) can be described as follows: find $\phi_{h}(t) \in C^{0}\left([0, \bar{T}] ; V_{h}\right)$, such that

$$
\phi_{h}(t)+\int_{0}^{t} p(t, \tau) P_{h} B(t, \tau) \phi_{h}(\tau) d \tau=P_{h} f(t), \quad t \in[0, \bar{T}] .
$$

For concrete $V, V_{h}$, and $P_{h},(3.1)$ may be the Galerkin method, the Lagrange collocation method, the spline collocation method (see [2]), and the Galerkin-Petrov method, or some other methods. For example, choose $V=H^{0}(\Gamma), V_{h}(0<h<1)$ to be a family of subspaces of $H^{0}(\Gamma)$ and $P_{h}$ to be the corresponding orthogonal projections. Then (3.2) is called the semi-discrete Galerkin method. In the twodimensional case, i.e. $\Gamma$ is a closed curve, it is easy to define $V_{h}$ to be the space of continuous piecewise polynomial functions of Lagrange type for a partition of $\Gamma$. For $\Gamma$ a closed surface, it is more difficult to construct $V_{h}$ such that $V_{h} \subset C^{0}(\Gamma)$. But we can define $V_{h}$ to be the space of piecewise continuous polynomial functions of Lagrange type and require that the elements of $V_{h}$ are continuous at all nodes of the partition. We refer to [16] for a construction of subspaces $V_{h}$ of $C^{0}(\Gamma)$. Let $P_{h}$ be the interpolation operator from $C^{0}(\Gamma)$ to $V_{h}$. It follows from Remark 2.1 that (3.2) is valid for any case of $V_{h}$, and we call (3.2) the semi-discrete Lagrange collocation method. 
We now consider discretizing equation (3.2). Since (3.2) is a Volterra integral system with a weakly singular kernel $p(t, \tau)$, we shall adopt the product integration method (see [3], [13]). For positive integer $N_{t}$, let $r=\bar{T} / N_{t}, t_{n}=n r$ $\left(n=1,2, \ldots, N_{t}\right)$. Suppose there are product integration formulas

$$
\int_{0}^{t_{n}} p\left(t_{n}, \tau\right) B\left(t_{n}, \tau\right) \phi(\tau) d \tau=\sum_{j=0}^{n} w_{n j} B\left(t_{n}, t_{j}\right) \phi\left(t_{j}\right)+\delta_{n}(\phi), \quad n=n_{0}, \ldots, N_{t},
$$

where $n_{0}$ is a given positive integer, $B\left(t_{n}, t_{n}\right) \phi\left(t_{n}\right)=\lim _{t \rightarrow t_{n}^{-}} B\left(t_{n}, t\right) \phi\left(t_{n}\right)$. Then the step by step method for (3.1) is described as follows: find $\phi_{h}^{n} \in V_{h}\left(n=n_{0}, \ldots, N_{t}\right)$ such that

$$
\phi_{h}^{n}+\sum_{j=0}^{n} w_{n j} P_{h} B\left(t_{n}, t_{j}\right) \phi_{h}^{j}=P_{h} f\left(t_{n}\right),
$$

where $\phi_{h}^{0}, \ldots, \phi_{h}^{n_{0}-1}$ are the approximate values of $\phi\left(t_{0}\right), \ldots, \phi\left(t_{n_{0}-1}\right)$. When $w_{n n}$ are small enough, $\phi_{h}^{n}\left(n=n_{0}, \ldots, N_{t}\right)$ can be found step by step from system (3.4). It is not difficult to observe that the precision of the rule (3.3) is one of the main factors which determine the convergence rate of the method (3.4). For raising the convergence order for the time step $r$, block by block methods (see [3], [13]) can be employed, but we do not elaborate them here.

It is easy to construct various step by step methods by choosing $V, V_{h}, P_{h}$, and the quadrature rule (3.3). For instance, we use the product Euler rule or the product trapezoidal rule in (3.3) and the Galerkin method or the Lagrange collocation method in (3.2). Then (3.4) is called the Euler-Galerkin method, the trapezoidal Galerkin method, the Euler-Lagrange collocation method, or the trapezoidal collocation method. In addition, we can define the Euler spline collocation method, the Euler-Petrov-Galerkin method, etc.

We conclude this section with some remarks on computational aspects. We first consider how to evaluate $P_{h} B\left(t_{n}, t_{j}\right) \phi_{h}^{j}$ in (3.4). It is sufficient to compute $P_{h} B\left(t_{n}, t_{j}\right) \chi$ for a basis function $\chi(x)$. When $j=n$, we have

$$
B\left(t_{n}, t_{n}\right) \chi(x)=\lim _{t \rightarrow t_{n}^{-}} B\left(t_{n}, t\right) \chi(x)=\Lambda(x) \chi(x), \quad x \in \Gamma,
$$

where

$$
\begin{aligned}
& \Lambda(x)=-\int_{R^{n-1}}(\pi)^{n / 2} D^{2} \sigma(\xi)\left(A^{-1}(\xi) \eta\right)^{2} \cdot n(x) \exp \left(-|\eta|^{2}\right) d \eta \\
& A(\xi)=\left([D \sigma(\xi)]^{T} D \sigma(\xi)\right)^{1 / 2},
\end{aligned}
$$

$y=\sigma(\zeta)\left(\zeta \in\left\{\eta \in R^{n-1}:|\eta-\xi|<\delta\right\}, \xi \in R^{n-1}, x=\sigma(\xi)\right)$ is the local coordinate of a neighbourhood of $x \in \Gamma$. It needs to be pointed out that $\Lambda(x)$ is independent of the choice of $\sigma$. When $j \neq n$, the kernel of $B\left(t_{n}, t_{j}\right)$ is smooth. Noting that the support of $\chi$ is quite small, we can reasonably compute $P_{h} B\left(t_{n}, t_{j}\right) \chi$ $(j=0,1, \ldots, n)$ by using numerical quadrature of low order. Now we consider the computation of $P_{h} f\left(t_{n}\right)$ in (3.4) and $w(t, x)$ in the integral expression of the solution $u(t, x)$ of $(2.1)-(2.3)$. They all contain two integral terms. Since the first integral terms have smooth kernels, we can compute them by using the standard numerical integrations. If we decompose the $\Omega$ into $D_{x, \delta}=\{y \in \Omega:|y-x|<\delta\}$ and $\Omega_{x, \delta}=\Omega \backslash \bar{D}_{x, \delta}$ ( $\delta$ is a small positive number , then each second integral term can 
be evaluated through computing a proper integration on $\Omega_{x, \delta}$ and an integration with a weakly singular kernel on $D_{x, \delta}$. Using coordinate transformations, we can simplify the integral on $D_{x, \delta}$ into an easily computed one.

\section{Error estimate}

Using the discrete Gronwall inequality (see [12], [13]) and Theorem 2.1, we can prove the following error estimate for (3.4).

Theorem 4.1. Suppose that $\phi \in C^{0}([0, \bar{T}] ; V)$ and $P_{h}(0<h<1)$ are uniformly bounded in $h$. Let $M_{1}=\sup _{0<h<1}\left\|P_{h}\right\|$, where $\|\cdot\|$ denotes the norm of space $H^{s}(\Gamma)$ or $C^{0}(\Gamma)$. Let $M_{2}$ be the positive constant in Theorem 2.1. Suppose that there exists a positive integer $m$ such that

$$
M_{1} M_{2} \sum_{j=j_{m-1}}^{\min (n, j)}\left|w_{n j}\right| \leq \alpha<1, \quad M_{1} M_{2} \sum_{j=j_{\nu}}^{\min \left(n, j_{\nu+1}-1\right)}\left|w_{n j}\right| \leq \alpha<1,
$$

for $n=n_{0}, \ldots, N_{t}, \nu=0,1, \ldots, m-1$, where $j=\left[\nu N_{t} / m\right], \nu=0,1, \ldots, m$, $j_{0} \leq n_{0} \leq j_{1}$. Then $\phi_{h}^{n}\left(n=n_{0}, \ldots, N_{t}\right)$ can be found from equation (3.4) step by step. In addition, the error estimate

$$
\begin{aligned}
& \left\|\phi\left(t_{n}\right)-\phi_{h}^{n}\right\| \\
& \quad \leq(1-\alpha)^{-m-1}\left[\max _{1 \leq n \leq N_{t}}\left(\left\|\left(I-P_{h}\right) \phi\left(t_{n}\right)\right\|+M_{1}\left\|\delta_{n}(\phi)\right\|\right)+\sum_{j=0}^{n_{0}-1}\left\|\phi\left(t_{j}\right)-\phi_{h}^{j}\right\|\right]
\end{aligned}
$$

holds for $n=n_{0}, \ldots, N_{t}$.

Proof. Let $e_{h}^{n}=\phi\left(t_{n}\right)-\phi_{h}^{n}$. Then, from (3.1), (3.4) and (3.5),

$$
e_{h}^{n}+\sum_{j=0}^{n} w_{n j} P_{h} B\left(t_{n}, t_{j}\right) e_{h}^{j}=\left(I-P_{h}\right) \phi\left(t_{n}\right)-P_{h} \delta_{n}(\phi) .
$$

Thus, from Theorem 2.1,

$$
\left\|e_{h}^{n}\right\| \leq M_{1} M_{2} \sum_{j=0}^{n}\left|w_{n j}\right|\left\|e_{h}^{j}\right\|+\left\|\left(I-P_{h}\right) \phi\left(t_{n}\right)\right\|+M_{1}\left\|\delta_{n}(\phi)\right\| .
$$

The estimate follows from the discrete Gronwall inequality.

Assume that $V_{h}$ has the approximation property:

$$
\left\|v-P_{h} v\right\|_{0} \leq C h^{s}\|v\|_{s}, \quad \forall v \in H^{s}(\Gamma),
$$

for the Galerkin method or

$$
\left\|v-P_{h} v\right\|_{0, \infty} \leq C h^{l}\|v\|_{l, \infty}, \quad \forall v \in C^{l}(\Gamma),
$$

for the collocation method, where $0 \leq s \leq k$ or $0 \leq l \leq k, k$ is a positive integer. Then we immediately have the following corollaries by using Theorem 2.1 and 4.1.

Corollary 4.1. Let $\phi$, the solution of equation $(3.1)$, belong to $C^{1}\left([0, \bar{T}] ; H^{0}(\Gamma)\right) \bigcap$ $C^{0}\left([0, \bar{T}] ; H^{m}(\Gamma)\right)$. Then for small enough $r$, the error estimate for the EulerGalerkin method

$$
\left\|\phi\left(t_{n}\right)-\phi_{h}^{n}\right\|_{0} \leq C\left[\left\|\phi\left(t_{0}\right)-\phi_{h}^{0}\right\|_{0}+h^{s}\|\phi\|_{s, 0, \infty}+\varepsilon_{n} r\left(\|\phi\|_{0,1, \infty}+\|\phi\|_{2,0, \infty}\right)\right]
$$

holds for $n=1,2, \ldots, N_{t}, s=\min (m, k)$, where $\varepsilon_{n}=t_{n}^{1 / 2}$ or $t_{n}$ when $p(t, \tau)=$ $(t-\tau)^{-1 / 2}$ or $2 t\left(t^{2}-\tau^{2}\right)^{-1 / 2}, 2 \tau\left(t^{2}-\tau^{2}\right)^{-1 / 2}$. 
Proof. From Theorem 4.1, it is enough to estimate $\left\|\delta_{n}(\phi)\right\|_{0}$. By Theorem 2.1, we have

$$
\left\|B\left(t_{n}, \tau\right) \phi(\tau)-B\left(t_{n}, t_{j}\right) \phi\left(t_{j}\right)\right\|_{0} \leq C r\|\phi\|_{2,0, \infty}
$$

for $t_{j} \leq \tau \leq t_{j+1}$. Hence,

$$
\begin{aligned}
\| B\left(t_{n}, \tau\right) \phi(\tau) & -B\left(t_{n}, t_{j}\right) \phi\left(t_{j}\right)\left\|_{0} \leq\right\| B\left(t_{n}, \tau\right) \phi\left(t_{j}\right)-B\left(t_{n}, t_{j}\right) \phi\left(t_{j}\right) \|_{0} \\
& +\left\|B\left(t_{n}, \tau\right)\left(\phi(\tau)-\phi\left(t_{j}\right)\right)\right\|_{0} \leq C r\left(\|\phi\|_{2,0, \infty}+\|\phi\|_{0,1, \infty}\right) .
\end{aligned}
$$

Thus,

$$
\begin{aligned}
\left\|\delta_{n}(\phi)\right\|_{0} & =\left\|\sum_{j=0}^{n-1} \int_{t_{j}}^{t_{j+1}} p\left(t_{n}, \tau\right)\left(B\left(t_{n}, \tau\right) \phi(\tau)-B\left(t_{n}, t_{j}\right) \phi\left(t_{j}\right)\right)\right\|_{0} \\
& \leq C r \int_{t_{0}}^{t_{n}} p\left(t_{n}, \tau\right) d \tau\left(\|\phi\|_{2,0, \infty}+\|\phi\|_{0,1, \infty}\right) \\
& \leq C \varepsilon_{n} r\left(\|\phi\|_{2,0, \infty}+\|\phi\|_{0,1, \infty}\right) .
\end{aligned}
$$

Analogously we can establish the error estimates of the other methods.

Corollary 4.2. Let $\phi$, the solution of equation (3.1), belong to $C^{1}\left([0, \bar{T}] ; C^{0}(\Gamma)\right) \cap$ $C^{0}\left([0, \bar{T}] ; C^{m}(\Gamma)\right)$. Then for small enough $r$, the error estimate for the EulerLagrange collocation method

$$
\left\|\phi\left(t_{n}\right)-\phi_{h}^{n}\right\|_{0, \infty} \leq C\left[\left\|\phi\left(t_{0}\right)-\phi_{h}^{0}\right\|_{0, \infty}+h^{l}\|\phi\|_{l, 0}+\varepsilon_{n} r\left(\|\phi\|_{0,1}+\|\phi\|_{2,0}\right)\right]
$$

holds for $n=1,2, \ldots, N_{t}$, where $l=\min (m, k), \varepsilon_{n}$ is as in Corollary 4.1.

Corollary 4.3. Let $\phi$, the solution of equation $(3.1)$, belong to $C^{0}\left([0, \bar{T}] ; H^{m}(\Gamma)\right) \bigcap$ $C^{1}\left([0, \bar{T}] ; H^{2}(\Gamma)\right) \cap C^{2}\left([0, \bar{T}] ; H^{0}(\Gamma)\right)$. Then for small enough $r$, the error estimate for the trapezoidal Galerkin method

$$
\begin{aligned}
\left\|\phi\left(t_{n}\right)-\phi_{h}^{n}\right\|_{0} \leq C\left[\left\|\phi\left(t_{0}\right)-\phi_{h}^{0}\right\|_{0}\right. & +h^{s}\|\phi\|_{s, 0, \infty} \\
& \left.+\varepsilon_{n} r^{2}\left(\|\phi\|_{4,0, \infty}+\|\phi\|_{2,1, \infty}+\|\phi\|_{0,2, \infty}\right)\right]
\end{aligned}
$$

holds for $n=1,2, \ldots, N_{t}, s=\min (m, k), \varepsilon_{n}$ is as in Corollary 4.1.

Corollary 4.4. Let $\phi$, the solution of equation (3.1), belong to $C^{0}\left([0, \bar{T}] ; C^{m}(\Gamma)\right) \bigcap$ $C^{1}\left([0, \bar{T}] ; C^{2}(\Gamma)\right) \cap C^{2}\left([0, \bar{T}] ; C^{0}(\Gamma)\right)$. Then for small enough $r$, the error estimate for the trapezoidal Lagrange collocation method

$$
\begin{aligned}
\left\|\phi\left(t_{n}\right)-\phi_{h}^{n}\right\|_{0, \infty} \leq C\left[\left\|\phi\left(t_{0}\right)-\phi_{h}^{0}\right\|_{0, \infty}\right. & +h^{l}\|\phi\|_{l, 0} \\
& \left.+\varepsilon_{n} r^{2}\left(\|\phi\|_{4,0}+\|\phi\|_{2,1}+\|\phi\|_{0,2}\right)\right]
\end{aligned}
$$

holds for $n=1,2, \ldots, N_{t}$, where $l=\min (m, k), \varepsilon_{n}$ is as in Corollary 4.1.

Remark 4.1. From Theorem 4.1, we can obtain that the convergence rate of the method in [15] is $O\left(r+h^{k+1}\right)$, which was conjectured in [15]. 
TABLE 5.1. Maximum errors as a function of $r$ and $t$

\begin{tabular}{|c|c|c|c|c|}
\hline $\mathrm{t}$ & \multicolumn{2}{|c|}{3.0} & \multicolumn{2}{c|}{5.0} \\
\hline $\mathrm{r}$ & ME & Rate & ME & Rate \\
\hline $1 / 4$ & $1.122236 \mathrm{E}-03$ & & $1.760125 \mathrm{E}-03$ & \\
\hline $1 / 8$ & $5.182028 \mathrm{E}-04$ & 1.1147 & $6.786585 \mathrm{E}-04$ & 1.3749 \\
\hline $1 / 16$ & $8.559227 \mathrm{E}-05$ & 2.5979 & $1.029372 \mathrm{E}-04$ & 2.7209 \\
\hline $1 / 32$ & $2.878904 \mathrm{E}-05$ & 1.5719 & $3.659725 \mathrm{E}-05$ & 1.4919 \\
\hline $1 / 64$ & $5.960464 \mathrm{E}-06$ & 2.2720 & $6.794930 \mathrm{E}-06$ & 2.4292 \\
\hline
\end{tabular}

TABle 5.2. ME as a function of $h$ and $r$ at $t=5.0$

\begin{tabular}{|c|c|c|c|}
\hline $\mathrm{r}$ & $\mathrm{h}=1 / 4$ & $\mathrm{~h}=1 / 8$ & $\mathrm{~h}=1 / 16$ \\
\hline $1 / 4$ & $2.339792 \mathrm{E}-02$ & $7.428646 \mathrm{E}-03$ & $1.580477 \mathrm{E}-03$ \\
\hline $1 / 8$ & $9.186625 \mathrm{E}-03$ & $5.038381 \mathrm{E}-03$ & $1.470089 \mathrm{E}-03$ \\
\hline $1 / 16$ & $7.557869 \mathrm{E}-04$ & $2.408266 \mathrm{E}-03$ & $7.539988 \mathrm{E}-04$ \\
\hline $1 / 32$ & $3.575206 \mathrm{E}-03$ & $4.023314 \mathrm{E}-04$ & $3.551245 \mathrm{E}-04$ \\
\hline $1 / 64$ & $5.920649 \mathrm{E}-03$ & $8.140802 \mathrm{E}-04$ & $4.255772 \mathrm{E}-05$ \\
\hline
\end{tabular}

\section{NumERICAL EXAMPLES}

We present some numerical results for the trapezoidal Lagrange collocation method (TLCM). In all examples, $\Omega$ is the unit disk and $\Gamma=\left\{x \in R^{2}:|x|=1\right\}$. The parametric representation of $\Gamma$ is $x=(\cos 2 \pi \theta, \sin 2 \pi \theta)(0 \leq \theta \leq 1)$. $\Gamma$ is decomposed into $N_{x}$ segmental arcs. $V_{h}$ consists of corresponding piecewise linear functions, where $h=1 / N_{x}$. All calculations were performed on a Great Wall 386 computer in single precision.

Example 5.1. We investigate TLCM for (2.6). First, let the exact solution of (2.6) be $\varphi(t, x)=1.0$. Note that $\varphi$ is independent of the $\theta$ coordinate. This is reflected in numerical results; therefore, we can observe the rates of convergence with respect to the time step $r$ for a fixed spatial step $h$. From Table 5.1, we find the average rates of convergence are 1.889 and 1.964 at $t=3.0,5.0$, respectively, where $h=1 / 8$ and ME is the maximum norm of the error.

Second, let the exact solution of $(2.6)$ be $\varphi(t, x)=\cos 2 \pi \theta$. In Table 5.2, we display the maximum errors as a function of the time step $r$ and the spatial step $h$ at $t=5.0$. These results indicate that the MEs are smallest when $r=h / 4$. This implies that the time step length should be chosen smaller than the space step length. The same phenomena occur in the next example. In Table 5.3, we give the rates of convergence for each pair of spatial steps (here we choose $r=h / 4$ ). The results show quadratic convergence, which is consistent with the expected rate $O\left(h^{2}+r^{2}\right)$.

Example 5.2. Let the solution of (2.4) be $\varphi(t, x)=t^{-1 / 2} \cos 2 \pi \theta$. In this example, we find $\psi(t)=t \varphi\left(t^{2}, x\right)$ from the equation (2.7) by using TLCM. Table 5.4 shows that the maximum errors become small as the time step decreases for a fixed spatial step. In Table 5.5, we set $r=h / 4$ and present the rates of convergence for two pairs of spatial steps. The results show that the rates are faster than the theoretical rates $O\left(h^{2}+r^{2}\right)$. 
TABLE 5.3. The rate of convergence

\begin{tabular}{|c|c|c|}
\hline $\mathrm{t}$ & $\mathrm{h}=1 / 4,1 / 8$ & $\mathrm{~h}=1 / 8,1 / 16$ \\
\hline 1.0 & 1.9252 & 1.9145 \\
\hline 1.5 & 1.9909 & 1.8930 \\
\hline 2.0 & 2.0602 & 1.8456 \\
\hline 2.5 & 2.1474 & 1.7652 \\
\hline 3.0 & 2.2875 & 1.6406 \\
\hline
\end{tabular}

TABLE 5.4. ME as a function of $h$ and $r$ at $t=5.0$

\begin{tabular}{|c|c|c|c|}
\hline $\mathrm{r}$ & $\mathrm{h}=1 / 8$ & $\mathrm{~h}=1 / 16$ & $\mathrm{~h}=1 / 32$ \\
\hline $1 / 8$ & $3.097606 \mathrm{E}-01$ & $2.967817 \mathrm{E}-01$ & $2.935145 \mathrm{E}-01$ \\
\hline $1 / 16$ & $7.920551 \mathrm{E}-02$ & $6.844246 \mathrm{E}-02$ & $6.573260 \mathrm{E}-02$ \\
\hline $1 / 32$ & $1.350176 \mathrm{E}-02$ & $5.002975 \mathrm{E}-03$ & $2.866507 \mathrm{E}-03$ \\
\hline $1 / 64$ & $8.301258 \mathrm{E}-03$ & $2.146482 \mathrm{E}-03$ & $5.843639 \mathrm{E}-04$ \\
\hline
\end{tabular}

TABLE 5.5. The rates of convergence

\begin{tabular}{|c|c|c|}
\hline $\mathrm{t}$ & $\mathrm{h}=1 / 4,1 / 8$ & $\mathrm{~h}=1 / 8,1 / 16$ \\
\hline 2.0 & 2.5364 & 1.3891 \\
\hline 3.0 & 2.8014 & 4.3346 \\
\hline 4.0 & 3.0487 & 2.2154 \\
\hline 5.0 & 3.1448 & 2.6531 \\
\hline
\end{tabular}

Remark 5.1. We also observed the same numerical results in the experiments of solving (2.8) by using TLCM.

Example 5.3. In this example, we consider the approximation of the solution of (2.1)-(2.3). We first compute the approximations of $\varphi(t, x), t \varphi\left(t^{2}, x\right)$ and $\varphi\left(t^{2}, x\right)$ from (2.6), (2.7) and (2.8), respectively, and then we use the representation formula

$$
u(t, x)=\int_{0}^{t} \int_{\Gamma} G(t-\tau, x-y) \varphi(\tau, y) d s(y) d \tau+w(t, x), \quad(t, x) \in(0, T] \times \Omega
$$

or

$$
u(t, x)=2 \int_{0}^{\sqrt{t}} \int_{\Gamma} G\left(t-\tau^{2}, x-y\right) \tau \varphi\left(\tau^{2}, y\right) d s(y) d \tau+w(t, x), \quad(t, x) \in(0, T] \times \Omega
$$

to recover approximation to the solution of (2.1)-(2.3). The integrations in (5.1) and (5.2) are computed by using the trapezoidal rule. Recalling the observations made in Examples 5.1 and 5.2, we choose $r=h / 4$ in all examples.

Let the solution of $(2.1)-(2.3)$ be as follows:

$$
u(t, x)=\exp \left(-\left|x-x_{0}\right| / 4 t\right) / t, \quad t>0, x \in \Omega,
$$

where $x_{0}=(2,0)$. Note that $g(t, x)=b(t, x)=\frac{\partial u(t, x)}{\partial n(x)} \in C^{\infty}([0, \infty] \times \Gamma)$ and $\frac{\partial^{k} g(0, x)}{\partial t^{k}}=0\left(x \in \Gamma, k \in Z^{+}\right)$. It follows from Theorem 4.1 of [21] that $\varphi \in$ $C^{\infty}([0, \infty] \times \Gamma)$ and $\frac{\partial^{k} \varphi(0, x)}{\partial t^{k}}=0\left(x \in \Gamma, k \in Z^{+}\right)$. In Tables 5.6, 5.7, and 5.8 we display the relative errors at spatial points: $(\rho \cos \theta, \rho \sin \theta), \theta=0.0, \pi / 3$, 
TABLE 5.6. (2.6)-Relative errors for $h=1 / 16, r=1 / 64$

\begin{tabular}{|c|c|c|c|c|c|c|}
\hline$\rho$ & \multicolumn{2}{|c|}{0.4} & \multicolumn{2}{c|}{0.8} & \multicolumn{2}{c|}{0.9} \\
\hline $\mathrm{t}$ & $\theta=0.0$ & $\theta=\pi / 3.0$ & $\theta=0.0$ & $\theta=\pi / 3.0$ & $\theta=0.0$ & $\theta=\pi / 3.0$ \\
\hline 1.00 & $7.8545 \mathrm{E}-03$ & $1.9240 \mathrm{E}-03$ & $1.5063 \mathrm{E}-02$ & $5.2012 \mathrm{E}-03$ & $2.3323 \mathrm{E}-02$ & $1.2674 \mathrm{E}-02$ \\
\hline 4.00 & $1.2377 \mathrm{E}-03$ & $3.3090 \mathrm{E}-04$ & $2.7337 \mathrm{E}-03$ & $1.0670 \mathrm{E}-03$ & $5.3365 \mathrm{E}-03$ & $3.1666 \mathrm{E}-03$ \\
\hline 9.00 & $1.5695 \mathrm{E}-05$ & $3.9414 \mathrm{E}-04$ & $6.2017 \mathrm{E}-04$ & $1.2354 \mathrm{E}-03$ & $2.0804 \mathrm{E}-03$ & $2.4730 \mathrm{E}-03$ \\
\hline
\end{tabular}

TABLE 5.7. (2.7) -Relative errors for $h=1 / 16, r=1 / 64$

\begin{tabular}{|c|c|c|c|c|c|c|}
\hline$\rho$ & \multicolumn{2}{|c|}{0.4} & \multicolumn{2}{c|}{0.8} & \multicolumn{2}{c|}{0.9} \\
\hline $\mathrm{t}$ & $\theta=0.0$ & $\theta=\pi / 3.0$ & $\theta=0.0$ & $\theta=\pi / 3.0$ & $\theta=0.0$ & $\theta=\pi / 3.0$ \\
\hline 1.00 & $8.3564 \mathrm{E}-03$ & $2.2616 \mathrm{E}-03$ & $4.3554 \mathrm{E}-03$ & $8.0790 \mathrm{E}-03$ & $1.6939 \mathrm{E}-02$ & $5.1543 \mathrm{E}-03$ \\
\hline 4.00 & $1.4543 \mathrm{E}-03$ & $5.4921 \mathrm{E}-04$ & $2.2609 \mathrm{E}-03$ & $2.9718 \mathrm{E}-03$ & $6.3862 \mathrm{E}-03$ & $3.0470 \mathrm{E}-03$ \\
\hline 9.00 & $9.0003 \mathrm{E}-04$ & $9.8474 \mathrm{E}-04$ & $1.4544 \mathrm{E}-03$ & $1.7754 \mathrm{E}-03$ & $5.2465 \mathrm{E}-03$ & $3.4341 \mathrm{E}-03$ \\
\hline
\end{tabular}

TABLE 5.8. (2.8)-Relative errors for $h=1 / 16, r=1 / 64$

\begin{tabular}{|c|c|c|c|c|c|c|}
\hline$\rho$ & \multicolumn{2}{|c|}{0.4} & \multicolumn{2}{c|}{0.8} & \multicolumn{2}{c|}{0.9} \\
\hline $\mathrm{t}$ & $\theta=0.0$ & $\theta=\pi / 3.0$ & $\theta=0.0$ & $\theta=\pi / 3.0$ & $\theta=0.0$ & $\theta=\pi / 3.0$ \\
\hline 1.00 & $8.2792 \mathrm{E}-03$ & $2.1636 \mathrm{E}-03$ & $4.3083 \mathrm{E}-03$ & $8.1672 \mathrm{E}-03$ & $1.6897 \mathrm{E}-02$ & $5.2410 \mathrm{E}-03$ \\
\hline 4.00 & $1.2414 \mathrm{E}-03$ & $3.2656 \mathrm{E}-04$ & $2.4639 \mathrm{E}-03$ & $3.1935 \mathrm{E}-03$ & $6.1878 \mathrm{E}-03$ & $2.8280 \mathrm{E}-03$ \\
\hline 9.00 & $1.3839 \mathrm{E}-03$ & $1.4778 \mathrm{E}-03$ & $1.9264 \mathrm{E}-03$ & $2.2671 \mathrm{E}-03$ & $4.7834 \mathrm{E}-03$ & $2.9486 \mathrm{E}-03$ \\
\hline
\end{tabular}

$\rho=0.4,0.8,0.9$. The numbers $(2.6),(2.7)$ or $(2.8)$ in these tables mean that the corresponding integral equation is solved.

The results in Tables 5.6, 5.7, and 5.8 indicate that the convergence is uniform in $\Omega \times(0, T]$. In fact, let $\tilde{\phi}$ be the approximate solution of $(3.1)$ and $\tilde{u}(t, x)$ be defined by replacing $\phi$ with $\tilde{\phi}$ in (5.1) or (5.2), we then have

$$
|u(t, x)-\tilde{u}(t, x)| \leq C \sup _{\substack{0 \leq t \leq T \\ x \in \Gamma}}|\phi(t, x)-\tilde{\phi}(t, x)|, \quad \forall t>0, x \in \Omega .
$$

Moreover, in all of three examples we have the same convergence rates. We also observe that the computing times of three examples are almost equal, although the number of time steps for (2.6) is 3 times the number of time steps for (2.7) and (2.8). Recall that $t \varphi\left(t^{2}\right)$ is always suitably smooth, but $\varphi(t)$ and $\varphi\left(t^{2}\right)$ might be only continuous. Therefore, in the practical realization, we suggest first finding $t \varphi\left(t^{2}\right)$ from (2.7) and then recovering $u(t, x)$ by using (5.2).

\section{ACKNOWLEDGMEnTs}

The author is very grateful to Professor Li Ronghua for his constant encouragement and helpful discussions and to Professor W. L. Wendland for all of his help leading to improvements of the manuscript. Furthermore, he thanks the referee for detailed remarks and constructive suggestions. 


\section{REFERENCES}

1. D.N. Arnold and P.J. Noon, Coercivity of the single layer heat potential, J. Comp. Math. 8 (1989), 1-7. MR 90k:65186

2. D. N. Arnold and W.L. Wendland, On the asymptotic convergence of collocation methods, Math. Comp. 44 (1983), 349-381. MR 85h:65254

3. C.T.H. Baker, The Treatment of Integral Equations, Clarendon Press, 1977. MR 57:7079

4. C.A. Brebbia and D. Nardin, Solution of parabolic and hyperbolic time dependent problems using boundary elements, Comp. \& Maths with Aplls. 12b (1986), 1061-1072. MR 88f:65218

5. C.A. Brebbia and L.A. Wrobel, The solution of parabolic problems using the dual reciprocity boundary element, Advanced Boundary Element Methods (ed. by P.K. Cruse). MR 89b:65226

6. M. Costabel, Boundary integral operators for the heat equation, Integral Equation and Operator Theory 13 (1990), 498-552. MR 91j:35119

7. M. Costabel, K. Onishi and W.L. Wendland, A boundary element collocation method for the Neumann problem of the heat equation, Inverse and Ill-Posed Problems, Academic Press, Inc., 1987, pp. 369-384. MR 90k:65177

8. D. Curran and B.A. Lewis, Boundary element method for the solution of the transient diffusion equation in two dimensions, Appl. Math. Modeling 10 (1986), 107-113.

9. F. De Hoog and R. Weiss, On the solution of a Volterra integral equation with a weakly singular kernel, SIAM J. Math.Anal. 4 (1973), 561-573. MR 49:1818

10. A. Friedman, Partial Differential Equations of Parabolic Type, Prentice-Hall, 1964. MR 31:6062

11. Huang Mingyou, Finite Element Methods for Evolution Equations, Shanghai Press of Technology and Science, 1983.

12. Li Ronghua and Feng Guocheng, The Numerical Treatment for Differential Equations, The People's Education Press, 1980.

13. P. Linz, Analytical and Numerical Methods for Volterra Equations, SIAM Philadelphia, 1985. MR 86m:65163

14. J.L. Lions and E. Magenes, Non-homogeneous Boundary Value Problems and Applications, Vols. 1-2, Springer-Verlag, 1973. MR 50:2670; MR 50:2671

15. E.A. McIntyre, Jr., Boundary integral solutions for the heat equation, Math. Comp. 46 (1986), 71-79. MR 87h:65214

16. J.C. Nedelec, Curved finite element methods for the solution of integral singular equation in $R^{3}$, Comput. Methods Appl. Mech. Engrg. 8 (1976), 61-80. MR 56:13741

17. P.J. Noon, The single layer heat potential and Galerkin boundary element methods for the heat equation, Ph.D. thesis, University of Maryland, U.S.A., 1988

18. K. Onishi, Convergence in the boundary element method for heat equation, TRU Mathematics (Science University of Tokyo) 17 (1981), 213-225. MR 83a:65088

19. K. Onishi, Boundary element method for time dependent heat equation, Proceedings of the 1st Japan-China Symposium on Boundary Element Methods (ed. by M. Takana and Q. Du).

20. F. Sgallari, A weak formulation of boundary integral equations for time dependent problems, Appl. Math. Modeling 9 (1985), 295-301. MR 86h:65146

21. Yang Hongtao, A new analysis of Volterra-Fredholm boundary integral equations of the second kind, Northeast. Math. J. 13 (3) (1997), 325-334.

Department of Mathematics, Jilin University, Changchun, 130023, China 